\title{
La dynamique fluviale à l'épreuve des changements environnementaux : Quels enseignements applicables à l'aménagement des rivières?
}

\author{
J.P. Bravard \\ Département de Géographie, Université Lyon III \\ URA 260 du CNRS et PIREN Vallées Fluviales
}

\section{Introduction}

Un nouveau concept a fait son apparition dans le domaine de la gestion des cours d'eau. Il s'agit de l'« aménagement doux ", susceptible de concilier d'une part les objectifs habituels de l'aménagement qui sont la protection contre les crues, la défense des terres de fond de vallée et la satisfaction de fonctions de production (énergie, eau d'irrigation et eau potable, loisirs nautiques...), et dautre part le respect de l'environnement.

Trois approches convergentes conduisent en effet. depuis quelques années, à remettre en cause, de manière plus ou moins virulente. le bien-fondé des interventions de génie civil, quitte à empiéter sur ce qui fut, et qui reste, le territoire de l'ingénieur.

La première approche est celle de l'écologie scientifique dont les buts avoués sont de réhabiliter l'instabilité des rivières en laissant exprimer les potentialités de débordement et d'inondation au bénéfice des milieux humides des plaines alluviales ou en tolérant les processus d'érosion latérale qui sont garants d'un entretien naturel de la diversité des biotopes et des biocénoses ripicoles. Fait écho à cette position de principe le rapport du groupe de travail "Protection et gestion des plaines alluviales " présenté aux Assises nationales de l'eau qui se sont tenues a Paris en mars 1991; y est considérée comme de première importance la notion d'"espace de liberté " dans lequel seront "définitivement proscrits les aménagements visant à maintenir artificiellement le cours dans son lit actuel $"$.
La deuxième approche est celle de certains gestionnaires qui développent une argumentation de nature économique selon laquelle les coûts directs ou indirects d'aménagements ou d'usages de la ressource sont susceptibles de remettre en cause le bien-fondé de certaines politiques. Citons à titre d'exemple le fait que le prélèvement de 19,8 MT de graviers dans le lit de l'Arve entre 1950 et 1985 a rendu nécessaire un investissement de $126 \mathrm{MF}$ en ouvrages de défense contre les attaques de l'Arve, somme qui sera augmentée de $60 \mathrm{MF}$ à terme (X. BLANC et al., 1989) : ou bien le fait que la défense des terres agricoles de la basse vallée de l'Ain, érodées en moyenne à la vitesse de 1 à 2 hectares par an, a motivé la construction récente d'enrochements d'une valeur d'environ $6 \mathrm{MF}$ qui peuvent être comparés avec un prix moyen de l'hectare de terre agricole de $20000 \mathrm{~F}$.

L'approche qui sera développée ici s'inscrit dans ta longue durée et dans l'espace du bassin-versant. De manière traditionnelle, les partis d'aménagement retenus le long des rivières sont fondés sur la conception du " régime permanent " qui prend en considération un comportement moyen de la rivière à l'échelle de plusieurs décennies et la probabilité d'occurence d'épisodes de faible fréquence de retour. C"est le temps de l'ingénieur, qui postule un équilibre statique sur la période pendant laquelle l'aménagement doit remplir ses fonctions (M. NEwSON, 1986). Or des études récentes montrent que, sur ce même laps de temps, il n'y a pas d'equilibre, même dynamique (J. LEWIN et al.. 1988). Au contraire, la fin du $x x^{e}$ siècle s'inscrit dans une

\section{River dynamics resistant to environmental changes \\ What lessons can be learned from river developments?}

\begin{abstract}
Active rivers of France have been managed following the implicit hypothesis that fonctionning is stable on the long term. In fact, the major choices of management have been settled during the XIXth century, i.e. at the end of the so-called "Little Ice Age", a period which was caracterized by large floods and by a high rate of bedload transportation; the policy of Mountain Conservation, the river training schemes and the extraction of gravels have desequilibrated the fonctionning of many rivers which are presently endangered by a deficit of bedload. The maintenance of processes of coarse supply into rivers, the cessation of extractions and the restoration of mobile gravel beds are recommanded.
\end{abstract}




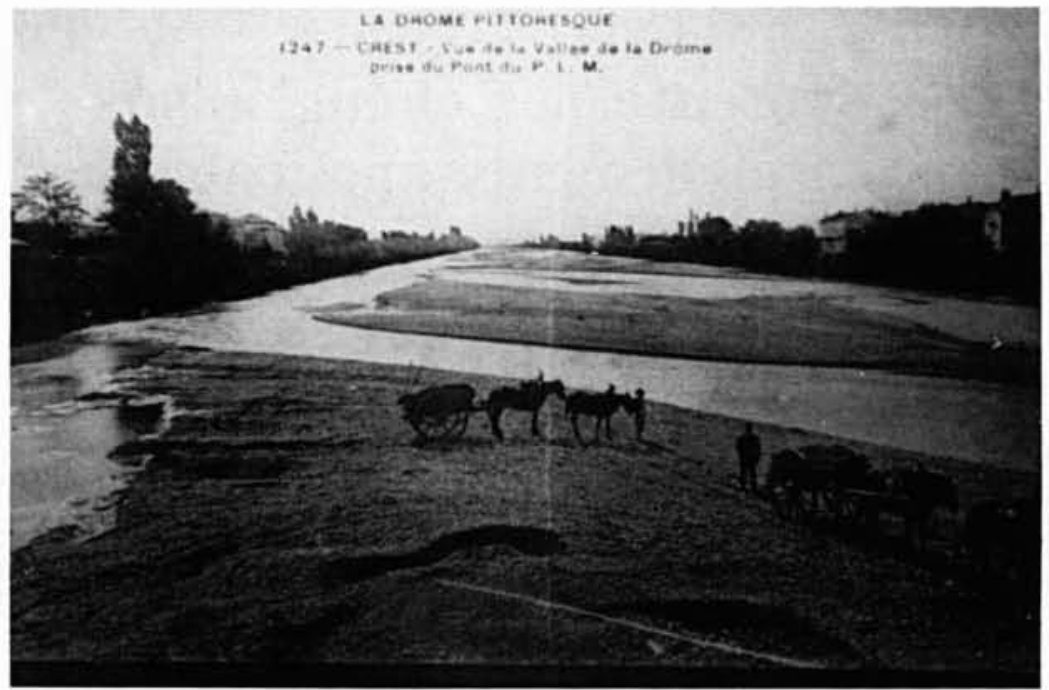

1. La Drôme à Crest vers 1910. Entre ses deux digues, la rivière tresse sur un épais remblaiement de cailloutis. Noter les extractions au premier plan.

2. La Drôme à Crest en 1987. Le lit est purgé des alluvions caillouteuses qui subsistent sur les bancs laterraux. Noter l'exhumation du bedrock qui forme des seuils naturels.

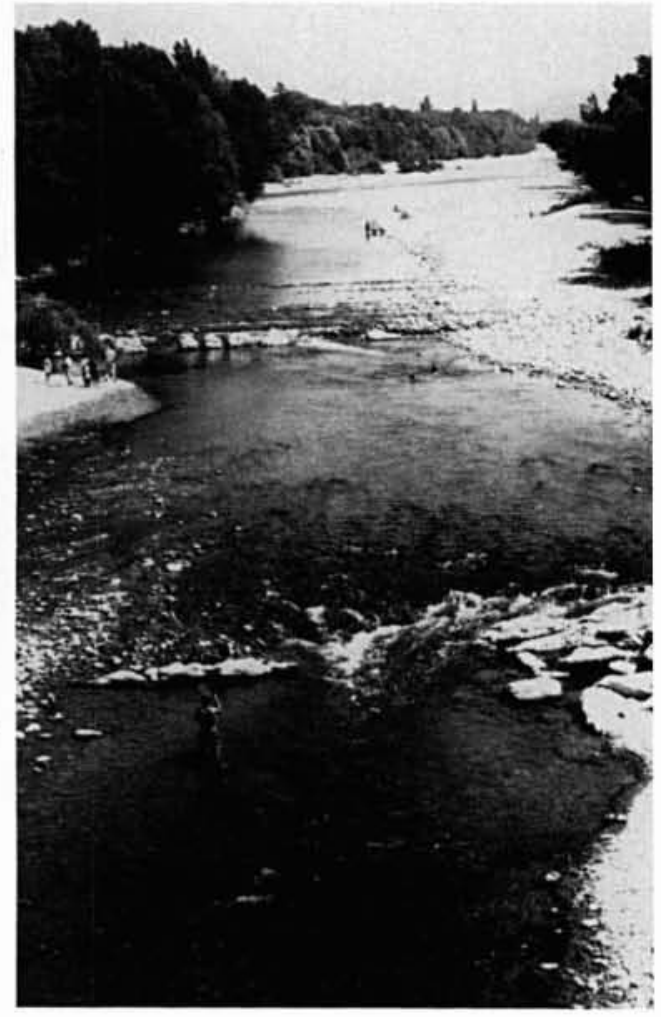

phase de mutation naturelle et de bouleversements d'origine anthropique qui perturbent de manière fondamentale le fonctionnement des rivières à dynamique active. L'enjeu est d'importance dans la mesure oủ les décideurs et gestionnaires sont demandeurs de prévision à long terme; la réponse passe sans doute par la prise en compte des fluctuations des hydrosystèmes fluviaux sur la longue durée et à l'échelle spatiale des bassin-versants.

\section{L'instabilité historique des rivières à fond mobile}

Pays de montagnes jeunes et de massifs anciens, la France est riche en cours d'eau à fonds mobiles, qu'ils soient de nature caillouteuse comme ceux des Alpes, des Pyrénées et de leur piedmont ou de la périphérie des Vosges et du Massif Central, ou qu'ils soient de nature plus sableuse comme l'Allier et la Loire. Les rivières les plus mobiles sont celles des zones dites d'érosion et de transit (S.A. Sснuмm, $1977)$ où des pentes encore fortes $(0,005$ et plus) et des pics de crue importants confèrent une puissance suffisante pour mettre en mouvement les matériaux grossiers et réaliser un ajustement de la géométrie aux variations des flux liquides et solides.

En revanche, en rivières de plaine, telles celles du Bassin Parisien, coulent sur des matériaux grossiers hérités des périodes froides et mis en place par des paléo-cours d'eau à forte compétence de charriage ; l'énergie disponible dans les conditions climatiques actuelles ne permet pas un ajustement rapide au changement. De fait, la plupart des enjeux actuels sont localisés sur les cours d'eau dynamiques intramontagnards ou situés à la périphérie des massifs, qui sont très sensibles aux fluctuations de charge et de débit sur la longue durée. Ce comportement a été quantifié au Royaume-Uni où les chenaux inactifs de plaine sont caractérisés par une puissance spécifique $\omega(=\rho g Q S / 1)$ comprise entre 1 et $60 \mathrm{~W} \cdot \mathrm{m}^{-2}$ avec une médiane de 15 (R. FERguson, 1981); ce résultat est à rapprocher de la mise en évidence d'un seuil de $35 \mathrm{~W} \cdot \mathrm{m}^{-2}$ au-dessous duquel les rivières ne sont pas capables de s'auto-ajuster en cas de perturbation liée à des aménagements (A. BROOKES, 1988).

L'étude géomorphologique des plaines alluviales confirme que celles-ci sont de très bons enregistreurs sédimentaires des fluctuations de la dynamique fluviale à l'Holocène. Durant les trois derniers millénaires, les cours d'eau à forte pente du bassin du Rhône ont connu deux phases majeures de forte activité, caractérisées par un fort transport de matériaux caillouto-sableux et par l'exhaussement de plusieurs mètres de l'espace de divagation de rivières à chenaux multiples; la première période, mal définie, correspond au $1^{\text {er }}$ Age du Fer, la deuxième à la fin du Moyen-Age et au Petit Age Glaciaire (1350-1880). Après l'Optimum Climatique Médiéval, période réputée chaude et sèche (E. Le Roi Ladurie, 1983 ; P. Alexandre, 1987) qui fut également une période d'incision des rivières car la charge était sans doute déficiente, une crise se produisit au milieu du $\mathrm{XIV}^{\mathrm{e}}$ siècle; une péjoration des volumes et du 
régime des précipitations provoqua une érosion accélérée des versants fragilisés par l'exploitation agricole. A partir de cette époque, des volumes considérables de matériaux furent injectés depuis la partie amont des bassins versants vers les cours d'eau de rang inférieur ; ils constituèrent des "mégaformes" (M. CHurCH \& D. JONES, 1982) progressant vers l'aval. Au XVIII ${ }^{\mathrm{e}}$ siècle, l'accumulation grossière en transit dans le Grésivaudan atteignait Grenoble alors que celle du Drac progressait déjà dans la plaine de Moirans après avoir franchi la cluse de Voreppe (J.P. Bravard, 1989 ; P.G. Salvador, 1990). Cette période, difficile pour les populations montagnardes et pour les riverains des grands fleuves, culmina avec les grandes crues du Rhône (1840 et 1856) et de la Durance (1886) qui furent d'autant plus dommageables qu'elles s'écoulèrent sur des chenaux et des bandes d'activité fluviale exhaussées sur leurs alluvions.

Cette crise morphodynamique - phénomène enregistré dans l'ensemble de l'Europe - est alors analysée puis combattue avec vigueur avant de faire place à un complet changement de situation (fig. 1 et 2 ). Plusieurs interventions ont conjugué leurs effets, mais de façon disjointe à l'échelle des bassins versants, de telle sorte que la prise de conscience n'en est que plus tardive:

2.1. Les endiguements longitudinaux destinés à la lutte contre les crues sont très anciens ; ils sont systématisés dès la fin du XVII ${ }^{e}$ siècle le long du Drac, à la fin du XVII" siècle sur le Haut-Rhône et dans la quasi-totalité des grandes vallées sous la Monarchie de Juillet. Leurs effets complexes sur le transit et le dépôt de la charge de fond sont tôt mis en évidence par les ingénieurs qui mettent l'accent sur les processus locaux d'exhaussement davantage que sur les processus d'incision qui commencent à apparaître (par exemple le basculement du canal de Miribel retracé par J. Winghart et J. ChABERT (1965). Ces aménagements ont eu un impact important à partir du moment où le tarissement des flux minéraux a produit ses effets.

2.2. Ce type d'intervention localisée qui vise à la défense immédiate des terres et de lieux habités fut doublée par une politique de réduction volontaire des flux hydriques et minéraux originaires des bassins versants montagnards. A tort ou à raison, les défrichements abusifs furent mis en accusation (P. Mougin, 1914 ; R. Nebort, 1983); dès le XVIII ${ }^{\circ}$ siècle, les autorités en tiraient des conséquences pratiques en Roussillon, telle, par une déclaration royale de 1777 , la prohibition des essartages sur les reliefs (B. Dessailly, 1990). Sous la Monarchie de Juillet, le reboisement des montagnes est considéré comme la panacée : «En outre des travaux effectués en lit de rivière, il y aurait d'autres mesures qui exerceraient une salutaire influence sur la navigabilité des cours d'eau naturels... je veux parler plus spécialement de la replantation des montagnes..." (M. Chevalier, 1838, Les intérêts matériels de la France, cité par B. Kalaora et A. SAvoye, 1986).

La loi de 1860 sur le reboisement des terrains de montagne, complétée par celle de 1882 qui met en œuvre une efficace correction torrentielle, va effectivement produire des effets contradictoires : d'une part contribuer efficace- ment à la réduction des crues et de l'érosion des versants, d'autre part réduire la charge grossière des rivières torrentielles et des grands cours d'eau (J.L. PeIRY, 1989).

2.3. Cette politique des Eaux et Forêts fut involontairement complétée par l'effet similaire des conséquences indirectes de l'exode rural qui toucha en premier lieu les régions d'altitude dès la fin du XIX ${ }^{\mathrm{e}}$ siècle. On sait que la crise des campagnes provoqua un recul des labours, une conversion à l'économie herbagère et un boisement spontané des pentes bien avant qu'on ne parle de la politique de la friche.

2.4. La fin du $\mathrm{XIX}^{\mathrm{e}}$ siècle inaugure l'ère de l'hydroélectricité et des réservoirs qui vont avoir pour effet de retenir une partie de la charge des cours d'eau. Dès 1930, on constate que le barrage du Motty, édifié en 1927 sur le Drac, s'engrave au rythme de $250000 \mathrm{~m}^{3} / \mathrm{an}$ (R. WALTHER, 1936). Dans d'autres régions sont construits des réservoirs d'alimentation en eau potable des grandes villes à l'amont desquels les bassins versants font également l'objet de précautions particulières.

2.5. Enfin la réduction des flux entrant dans le système fluvial, doublée d'un blocage accentué de leur transit, s'est accompagnée d'un prélèvement de plus en plus massif de sable ou gramulat qui dépasse de loin le volume des apports en provenance de l'amont à partir des années 1950-1960. Alors que la Loire transportait $0,2 \mathrm{MT} / \mathrm{an}$ de charge de fond avant cette période, les prélèvements s'élevaient à 10 MT en 1978 , soit $15000 \mathrm{~m}^{3}$ d'ablation moyenne par kilomètre linéaire de lit dans le Val orléanais et tourangeau (Y. Babonaux, 1986). Cette politique, rentable dans le court terme, présentait également l'avantage de réduire les risques d'inondation mais constitue une erreur de gestion fondamentale si l'on veut bien adopter une vision à long terme des processus.

Il est en effet clair que la politique de contrôle amont des flux hydriques et minéraux est concommitante d'une modification climatique encore mal connue; alors que la sortie du Petit Age Glaciaire aurait logiquement dû s'accompagner d'une lente « métamorphose " analogue à celle que les cours d'eau européens ont connu plusieurs fois à l'Holocène, les gestionnaires de la montagne et des cours d'eau n'ont cessé, depuis plus d'un siècle, d'agir comme si une abondante charge de fond était une constante du fonctionnement fluvial. D'une manière et de l'autre, et compte tenu des phénomènes d'impact amont et aval, la quasitotalité des branches du réseau hydrographique a été affectée, jusques et y compris sur les littoraux alimentés par les apports d'origine continentale. L'impact le plus immédiatement analysable est une incision accélérée des lits fluviaux qui compromet la stabilité d'ouvrages de génie civil, et fait peser des menaces sur la ressource en eau phréatique et sur la pérennité des milieux humides.

Dans les vallées où les cours d'eau sont capables de s'ajuster en réponse au jeu de variables externes (charge et débit), la notion de régime permanent ne suffit plus car le système est déséquilibré de manière durable; en effet, la période de "relaxation" dépassera probablement plusieurs décennies. 


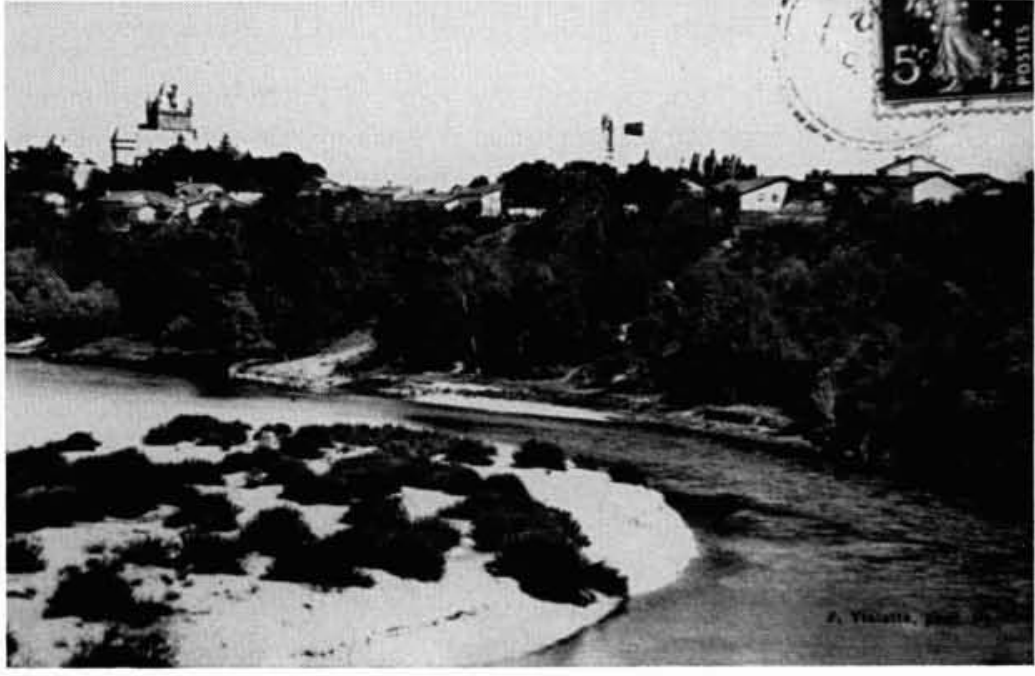

3.

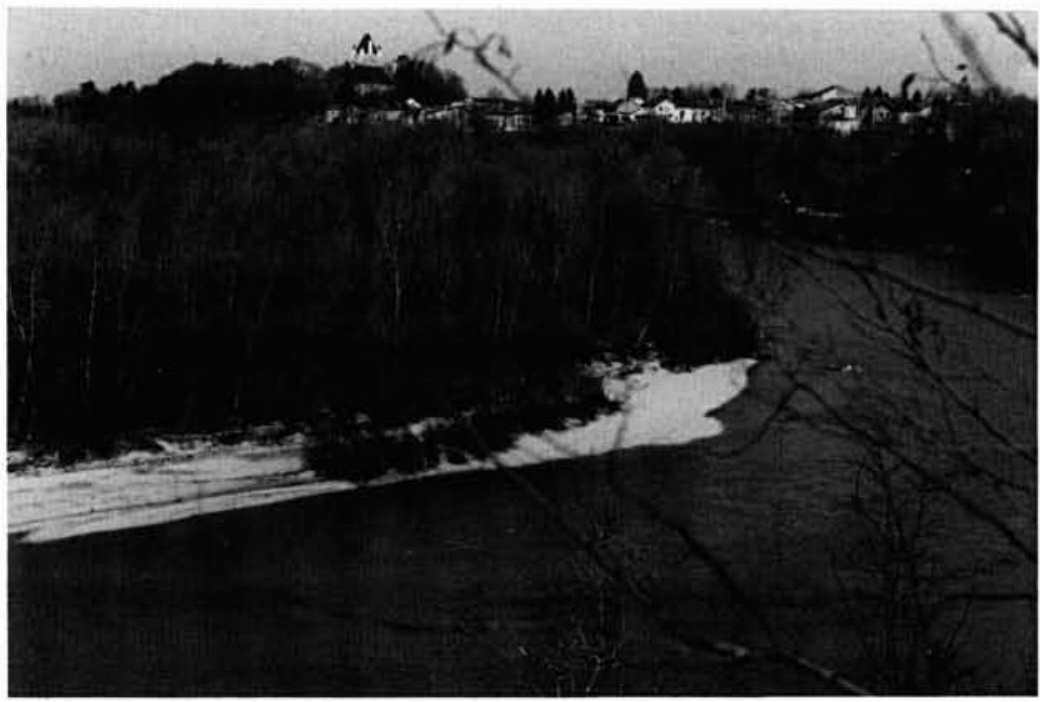

4.
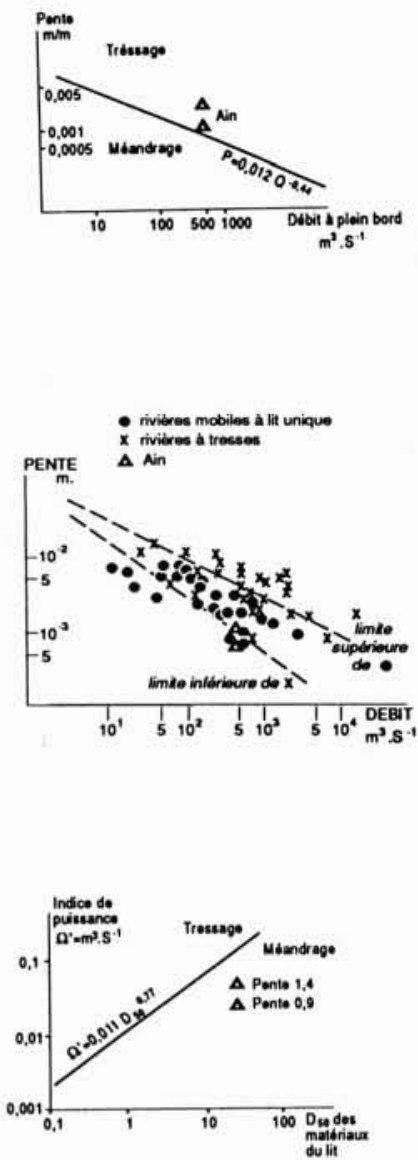

5.

3. L'Ain à Chazey en 1912. Un banc de convexité est colonisé par une saulaie pionnière.

4. L'Ain à Chazey en 1990. Le même banc de convexité est stabilisé par une forêt évoluée à base de peupliers noirs. Les transports de galets se sont fortement réduits.

5. Le style fluvial de la basse vallée de l'Ain : tressage ou méandrage?

1.1. L'Ain, rivière à lit unique, est partiellement une rivière à tresses d'après la relation de LEOPOLD et WOLMAN (1957). Cette approche ne prend pas en considération le diamètre des matériaux du lit, ni la charge.

1.2. La position limite de l'Ain est confirmée par le graphe de KeLLERHALS et BRAY (1986). Les cercles correspondent à des rivières citées dans la littérature; la dispersion des points s'explique par le jeu d'autres variables que la pente et le débit à plein bord.

1.3. La mise en relation du D50 des matériaux du lit et de l'indice de puissance (Pente $\times$ Débit à plein bord) selon la méthode de RICHARDS, classe l'Ain parmi les cours d'eau à lit unique : la réduction de la charge s'est probablement accompagnée d'une sélection des matériaux du lit au profit des gros éléments peu mobiles. Ceci expliquerait la disparition du tressage. 
Les enjeux d'une gestion "douce" sont donc, de ce point de vue, d'amortir les impacts négatifs du tarissement de la charge de fond. La basse rivière d'Ain fournit un exemple concret de cette situation.

\section{Etude de cas: La basse rivière d'Ain}

Cours d'eau long de 200 km, cet affluent du Rhône draine un bassin versant jurassien de $3500 \mathrm{~km}^{2}$; le module annuel est voisin de $120-130 \mathrm{~m}^{3} \cdot \mathrm{s}^{-1}$ au confluent. Jusqu'au début $\mathrm{du} \mathrm{xx}^{\mathrm{e}}$ siècle, le cours aval de l'Ain fut une section à chenaux multiples en réponse à une combinaison de variables favorables:

- une pente moyenne de $0,0013 \mathrm{~m} \cdot \mathrm{m}^{-1}$;

- des débits de pointe supérieurs à $2000 \mathrm{~m}^{3} \cdot \mathrm{s}^{-1}$ $\left(\mathrm{Q} 100=2400 \mathrm{~m}^{3} \cdot \mathrm{s}^{-1}\right)$;

- une charge de fond jamais évaluée, mais sans doute abondante.

Une étude historique de la rivière a montré que celle-ci a subi une métamorphose caractérisée par :

- la disparition du tressage depuis les années 1930 au profit d'un lit sinueux unique (J.P. BRAVARD, 1986) ;

- une forte réduction de la surface occupée par les bancs de galets non végétalisés, qui est passée de 279 ha en 1945 à 176 ha en 1965 avant de stabiliser (valeurs mesurées à l'étiage) :

- en parallèle, un développement accéléré de la forêt alluviale entre 1950 et 1980 , avec un pic dans les années 1960 (P. FAFOT et al., 1989). Les figures 3 et 4 donnent une vision imagée de cette évolution du paysage :

- une incision accélérée du lit mineur dans les tronçons non contrôlés par l'exhumation du bedrock molassique et de la moraine à blocs (J.P. BRAVARD et al., 1990).

L'application de diverses relations montre que l'Ain est dans une situation limite entre le méandrage et le tressage (fig. 5); la métamorphose observée est liée à la conjonction d'une série de facteurs naturels et anthropiques qui ont joué dans le même sens (J.P. BRAVARD et al., 1991) :

- la réduction de la charge de fond, qui est moins due à l'effet des réservoirs amont de Vouglans à Allement qu'à l'impact des digues et des ouvrages de franchissement de la rivière, dont l'effet a été de bloquer la recharge par érosion latérale :

- le boisement spontané de la bande d'activité fluviale, dû plus à une réduction du stress imposé par les crues qu'à l'abandon de l'affouage. En effet, le développement des ligneux a bénéficié de la réduction du transport de la charge de fond, avec d'autant plus de vigueur que les débits de pointe n'ont pas dépassé $1500 \mathrm{~m}^{3} \cdot \mathrm{s}^{-1}$ entre 1957 et 1990 (crue de période de retour égale à 7 ans à Pont de Chazey, calculée par SOGREAH sur la période 19131977).

L'incision de la rivière est la conséquence logique d'une telle évolution : en effet, il est admis qu'à débit égal, les rivières dont les berges sont boisées ont une largeur moyenne plus réduite. R. HEY (1986) a mis au point deux équations valables sur les cours d'eau britanniques à fond caillouteux :
$\mathrm{W}=2,34 \mathrm{Qb}^{0,50}$ si les berges sont boisées à plus de $50 \%$, $\mathrm{W}=2,73 \mathrm{Qb}^{0,50}$ si les berges sont boisées entre 5 et $50 \%$, (W est la largeur du cours d'eau en mètres, $\mathrm{Qb}$ le débit à plein bord).

La stricte application de ces équations au cours aval de l'Ain donnerait une largeur de 66 mètres dans le premier cas et de 77 mètres dans le second; en l'occurrence le boisement signifierait une réduction de largeur de $15 \%$ du lit à plein bord, changement confirmé par l'examen des photographies aériennes.

Toujours en conservant ces valeurs, le boisement contribue à une forte augmentation de la puissance spécifique du cours d'eau ; dans le premier cas elle est de 182 watts $\cdot \mathrm{m}^{-2}$, dans le second elle n'est que de 156 watts $\cdot \mathrm{m}^{-2}$. Ces valeurs, fortes pour un cours d'eau de plaine transportant des matériaux dont la médiane est voisine de $2,5 \mathrm{~cm}$, induisent une dissipation verticale de l'énergie et le creusement dont il a été question.

Une étude réalisée en 1990 par l'Agence de l'eau RhôneMéditerranée-Corse à la demande du Conseil régional de l'Ain a posé la question de la gestion à long terme de la rivière en fonction de cette situation de réduction des flux. L'intérêt actuel des biocénoses aquatiques et terrestres, déjà nettement réduit en quelques vingt ans, est fondé sur le maintien de la mobilité spatiale de l'Ain et sur le transit de la charge de fond (A.L. Roux, 1986). Une politique cohérente avec ses objectifs conduit :

1 - à déconseiller, sauf s'il s'agit de défendre des lieux habités, les enrochements latéraux de défense des terres agricoles en contact direct avec la rivière. C'est en effet par le jeu de l'érosion latérale des basses terrasses que la rivière pourra se recharger. Cette politique supposerait une juste indemnisation des pertes et dommages subis ;

2 - à promouvoir, sous bénéfice d'inventaire, le pavage du fond de façon à tenir le profil en long entre les rapides rocheux et à favoriser la dissipation latérale de l'énergie ; 3 - à proposer une coupe sélective de la ripisylve susceptible de permettre la dissipation latérale de l'énergie (CNR, 1987).

Il s'agit en somme d'assurer au cours d'eau une charge caillouteuse pendant les prochaines décennies, en favorisant le déstockage progressif des matériaux alluviaux présents dans le lit majeur. L'évolution récente a en effet conduit à déstocker de manière préférentielle les matériaux du lit mineur en voie de fixation.

\section{Un enjeu pour la gestion des rivières : Le maintien du transport solide}

Une approche historique a donc conduit à estimer qu'une politique trop strictement cantonnée à la maîtrise des crues et à l'exploitation de la ressource en granulats a mésestimé l'évolution historique des flux dans le système fluvial. Compte tenu de l'état de la question, une gestion prospective à l'échelle des bassins versants supposerait un ensemble de mesures coordonnées parmi lesquelles on peut citer : 


\section{Le maintien de processus d'injection de matériaux dans les rivières}

Le rapport de synthèse général du Colloque de Propriano (R.E. QUELENNEC, 1982) recommandait de réduire les transferts vers l'aval des sédiments issus des zones d'érosion concentrée les plus productives, dans la mesure où ils créent des nuisances pour les usages aval de la ressource en eau ; la recommandation qui en découle est d'augmenter l'efficacité des ouvrages de correction passive (ceux du Service de R.T.M.), par la valorisation des blocs et galets stockés.

Le curage des lits torrentiels sur les cônes de déjection fournit certes une ressource abondante, facile à prélever, mais son carctère systématique peut déséquilibrer le bilan sédimentaire d'une rivière. Sur les torrents du bassin de l'Arve les curages étaient peu développés avant 1950 ; dans un premier temps effectués après des crues fortement chargées pour assurer le déblaiement du chenal, ils sont devenus une véritable industrie à un point tel que la recharge de l'Arve est mise en péril (J.L. Peiry, 1989). Voici déjà dix ans, un grand forestier a mis en doute l'intérêt de continuer la politique de R.T.M. car les pentes sont abandonnées et car les extracteurs ont " trop d'appétit »; " estil dès lors justifié de créer à grands prix des ouvrages destinés à retenir ces matériaux en haut de gorges torrentielles inaccessibles ?... L'érosion en montagne apparaît donc bien - et le fait est nouveau - comme susceptible de gestion " (L. DE CRÉCY, 1982). Sans remettre en question les politiques de défense des terrains de montagne et de correction torrentielle qui sont nécessaires et efficaces, il paraît donc également raisonnable de tolérer l'injection de matériaux dans les rivières torrentielles qui ont le plus souffert de la gestion récente.

A ce propos, relevons le caractère ambigu du rapport du groupe de travail "Protection des milieux aquatiques" présenté aux Assises nationales de l'eau ; tolérant l'érosion des berges (p. 33), il met en avant le fait que «sur les terrains accidentés ou dégradés, le recours aux techniques de défense et de restauration des sols et de correction torrentielle est susceptible d'améliorer notablement le régime des eaux et de réduire les pollutions mécaniques dues à l'érosion " (p. 23). Sans doute, mais il conviendrait de distinguer ce qui relève de l'érosion des terres agricoles (essentiellement des matières en suspension), et d'autre part les processus qui assurent le renouvellement de la charge de fond. Contenue dans certaines limites, ce dernier type d'érosion n'est pas un facteur de pollution, mais est le seul moyen d'assurer une injection suffisante.

La fourniture de charge alluviale procède également de l'érosion latérale qui permet le progressif déstockage des plaines alluviales. L'ingénieur PARTIOT (1871) notait déjà que l'essentiel du charriage de la Loire était fourni par l'érosion des berges, alors que peu de matériaux venaient des ruisseaux. Pour sa part, J. TRICART (1962) a estimé que le charriage des alluvions grossières du Vidourle ne provient pas de l'érosion diffuse dans les Cévennes, mais du nettoyage du lit des torrents de versants et de quelques sapements de berge ; sur les cours d'eau de rang supérieur, une partie du transit est assurée par l'excavation naturelle des mouilles dans la nappe périglaciaire du fond de vallée.

Il paraît donc logique d'admettre que les cours d'eau à fond mobile souffrant d'un déficit de charge grossière soient assurés d'un minimum de mobilité latérale au sein d' " espaces de liberté ». Une telle politique rend nécessaire un consensus social difficile à acquérir, ou la maîtrise foncière des espaces riverains; en tout état de cause, elle signifie une réduction de la réalisation des enrochements de berge et, dans certains cas à étudier, leur enlèvement.

\section{Le maintien des matériaux dans le lit des rivières}

Une politique systématique, de nature conservationniste, devrait assurer la réalisation de trois objectifs :

- L'arrêt des extractions et un strict contrôle des curages dits d'entretien. A titre d'exemple, la politique de gestion des rivières menée en Haute-Savoie est sensée privilégier les solutions techniques visant à éliminer les zones d'engraissement local par une amélioration de la compétence du cours d'eau plutôt que par enlèvement des matériaux (X. BLANC et al., 1989);

- Le contrôle de l'incision par réalisation de seuils ou de pavages localisés; sans nuire à l'efficacité des ouvrages, un effort de recherche esthétique et une meilleure adaptation aux réalités écologiques des cours d'eau mériteraient d'être davantage pris en compte ;

- L'engraissement des lits des petites rivières naturellement ou artificiellement privées de leurs matériaux. A cet égard, la politique suivie par l'U.S. Department of Agriculture est novatrice: dans le but d'assurer le flottage des bois, les rivières de la chaîne côtière d'Oregon furent naturellement curées par les crues suite à l'enlèvement des embâcles à la fin du siècle dernier; la pose d'obstacles naturels (troncs, blocs) favorise le stockage de la charge de fond dans les lits et permet une relative restauration des conditions biologiques au profit du saumon.

En somme, une gestion douce des riivères ne peut faire l'économie d'une réflexion et d'actions menées à l'échelle des bassins versants et des plaines alluviales. L'évolution d'un lit fluvial conditionne le fonctionnement biologique de la rivière, le potentiel d'échange avec les nappes, le niveau de celles-ci, la conservation des zones humides qui leur sont liées, enfin la complexité des biocénoses terrestres.

Cette nécessaire ouverture spatiale de la gestion doit enfin se doubler d'une vision prospective du système fluvial, qui passe par une connaissance suffisante de l'évolution historique des flux et des ajustements. 


\section{Références bibliographiques}

Alexandre P. (1987). - Le climat en Europe au Moyen-Age. Contribution à l'histoire des variations climatiques de 1000 à 1425, d'après les sources narratives de l'Europe occidentalc. Paris, Ecole des Hautes Etudes en Sciences Sociales, Recherches d'histoire et de sciences sociales, $24: 828 \mathrm{p}$.

Babonaux Y. (1986). - L'espace ligérien, in M. Ferro Ed. : Une histoire de la Loire. Ramsay, pp. 37-69.

Blanc X., Pinteur F., Sanchis Th. (1989). - Conséquences de l'enfoncement du lit de l'Arve sur les berges et les ouvrages. Bilan général des transports solides sur le cours d'eau. La Houille Blanche, $\mathrm{n}^{\circ} 3-4$, pp. 226-230.

BRAVARD J.P. (1986). - La basse vallée de l'Ain: dynamique fluviale appliquée à l'écologie, in A.L. Roux (op. cit.), pp. 17-43.

BRAVARD J.P. (1989). - La métamorphose des rivières des Alpes françaises à la fin du Moyen-Age et à l'Epoque Moderne. in F. Petit, A. Laurant et A. Pissart (édit.). Sociëté Géographique de Liège, vol. 25, pp. 145-158.

Bravard J.P.. Franc O., Landon N.. Large J.L., Peiry J.L. (1990). - La basse vallée de l'Ain. Etude géomorphologique. Rapport dactylographié, $113 \mathrm{p}$.

Bravard J.P., Malavoi J.R., Amoros C. (1991), - L'Ain, ou la difficulté de gérer une rivière en cours de métamorphose. Actes de la Journée d'étude: "Rivières en crise: Saöne. Ain. Durance ", du 17-3-1990.

Brookes A. (1988). - Channelized rivers. Wiley \& Sons, Chichester, $326 \mathrm{p}$.

Church M., Jones D. (1982), - Channel bars in gravel-bed rivers, in R.D. Hey, J.C. Bathurst and C.R. Thor, Ed., Gravel Bed Rivers, Fluvial Processes, Engeniering and Management. Wiley \& Sons, pp. 291-338.

Compagnie Nationale du Rhône (1987). - Etude générale d'avant-projet de travaux d'aménagement de la riviére d'Ain entre le pont de Gévrieux et le Rhône. DTE 87, 437a.

CRECY L. de (1982). - Aspects techniques et socio-économiques des interactions entre les divers secteurs d'activité humaine et les processus sédimentaires dans le milieu amont. Montagne et domaine torrentiel, in : Rapports sur le colloque de Propriano, B.R.G.M., (2), III, pp. 4I-48.

Dessailly B. (1990). - Crues et inondations en Roussillon. Thèse de Géographie, Univ. Paris X, 352 p.

Fagot Ph., Gadiolet P., Magne M., Bravard J.P. (1989). Etude de dendrochronologie dans le lit majeur de l'Ain: la forêt alluviale comme descripteur d'un changement morphodynamique. Rev. Géogr. de Lyon, 4, pp. 213-223.

FERGUSON R. (1981). - Channel forms and channel changes, in
J. Lewin (Edit.) : British Rivers, G. Allen and Unwin, London, pp. 90-125.

HEy R. (1986). - River mechanics. Journal of the Institution of water engineering and Scientists, 40, 2, pp. 139-158.

Kalaora B., Savoye A. (1986), - La forêt pacifiée.

Le Roy Ladurie E. (1983). - Histoire du climat depuis l'An Mil. Flammarion, Paris, 2 vol., 287 et 254 pp.

Lewin J., MAcklin M., Newson M. (1988). - Regime theory and environmental change : irreconciliable concepts? Int. Conf. on River Regime. Hydraul. Research Ltd, J. Wiley, pp. $431-445$.

Mougin P. (1914), - Les torrents de la Savoie. Imp. Générale, Grenoble, 1215 .

NeBort R. (1983). - L'homme et l'érosion. Bull. Fac. Lettres et Sc. Humaines, Univ. Clermont-Ferrand II, nouv. Série, 17. $183 \mathrm{p}$.

NEWSON M. (1986). - River basin engineering. Fluvial Geomorphology. Journal of the Institution of Water Engineering and Scientists, 40, 4, pp. 307-324.

Partiot, 1871. Mémoire sur les sables de la Loire. Ann. Ponts et Chaussées, $5^{\text {e }}$ S., 1, pp. 233-292

Peiry J.L. (1989), - Les sédiments dans les torrents de l'Arve: discontinuité fonctionnelle et impacts de l'aménagement des bassins versants. La Houille Blanche, 3-4, pp. 205-211.

QuelenNec R.E. (1982). - Rapport de synthèse général, in : Rapports sur le colloque de Propriano, B.R.G.M.. (2), III, pp. 95-103.

Roux A.L. (1986). - Recherches interdisciplinaires sur les écosystèmes de la basse plaine de l'Ain (France). Doc. Cart. Ecol., XXIX, $166 \mathrm{p}$.

SALVAdOR, P.G. (1991). - Le thème de la métamorphose fluviale dans les plaines alluviales du Rhône et de l'Isère; bassin de Malville et ombilic de Moirans, Bas-Dauphiné. Thèse de doctorat, Université de Lyon 3, 498 p.

Sснимм S.A. (1977). - The fluvial system, J. Wiley and Sons, New York, $338 \mathrm{p}$.

Tricart J. (1962). - Les transports solides du Vidourle lors de la crue de l'automne 1958. $86^{\circ}$ Congrès Nat. Soc. Savantes, Montpellier, 1961, pp. 453-535.

WALther R. (1936). - Note sur l'engravement et le dégravement d'un bassin d'accumulation. Histoire du lac du Motty. Rev. Géogr. Alpine, pp. 315-328.

Winghart J., Chabert G. (1965). - Le Haut-Rhône à l'amont de Lyon : étude hydraulique de l'île de Miribel-Jonage. $\mathrm{La}$ Houille Blanche, 7, pp. 1-21. 
Theorie des Fleuves

Flanike IZI.

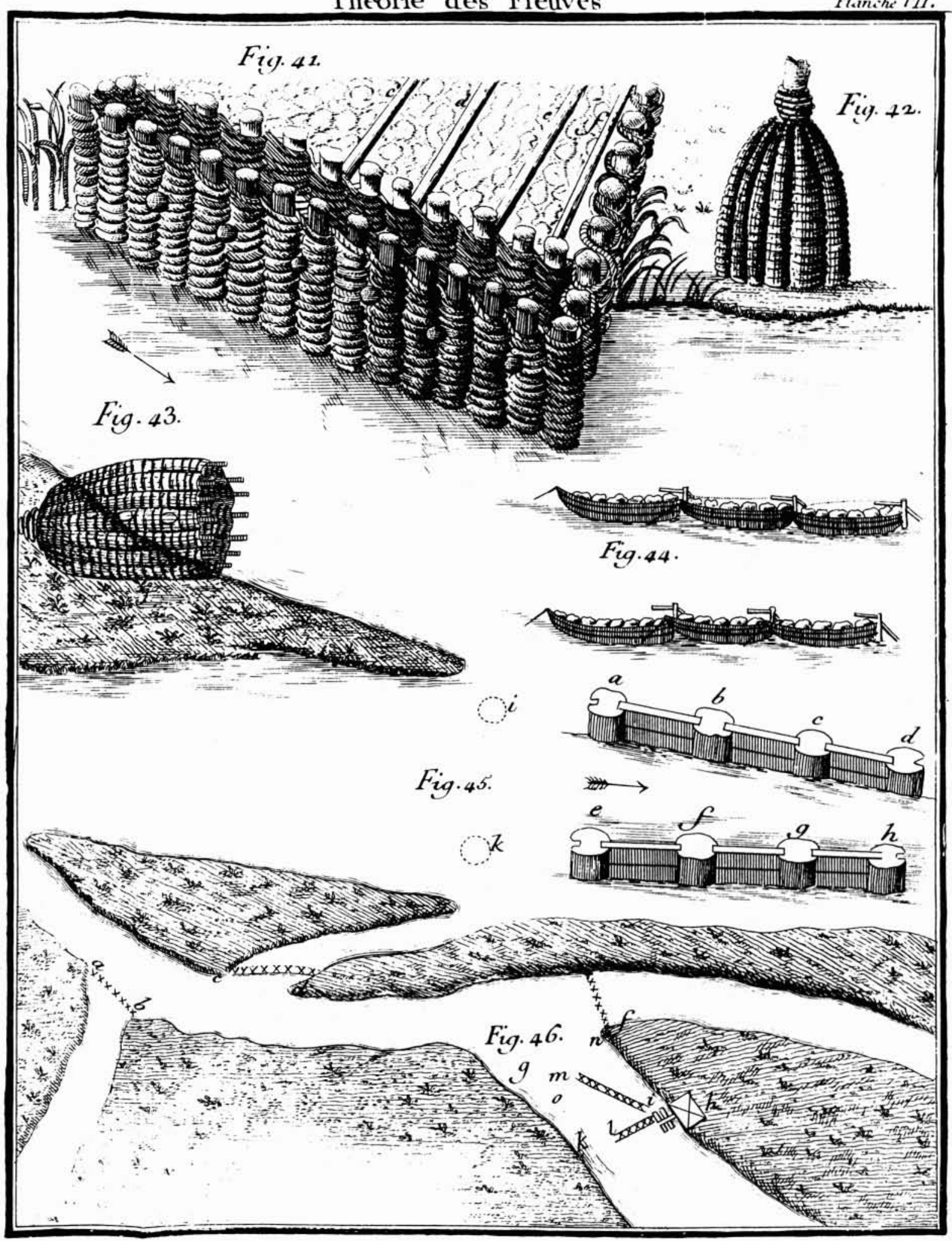

